\title{
Remote Hemorrhage after Burr Hole Drainage of Chronic Subdural Hematoma
}

\author{
Chang Hyeun Kim, Geun Sung Song, Young Ha Kim, Young Soo Kim, \\ Soon Ki Sung, Dong Wuk Son, and Sang Weon Lee \\ Department of Neurosurgery, Pusan National University Yangsan Hospital, Pusan National University School of Medicine, \\ Yangsan, Korea
}

\begin{abstract}
Chronic subdural hematoma (CSDH) and symptomatic subdural hygroma are common diseases that require neurosurgical management. Burr hole trephination is the most popular surgical treatment for CSDH and subdural hygroma because of a low recurrence rate and low morbidity compared with craniotomy with membranectomy, and twist-drill craniotomy. Many reports suggest that placing a catheter in the subdural space for drainage can further reduce the rate of recurrence; however, complications associated with this type of drainage include acute subdural hematoma, cortical injury, and infection. Remote hemorrhage due to overdrainage of cerebrospinal fluid (CSF) is another possible complication of burr hole trephination with catheter drainage that has rarely been reported. Here, we present 2 cases of remote hemorrhages following burr hole trephination with catheter drainage for the treatment of CSDH and symptomatic subdural hygroma. One patient developed intracerebral hemorrhage and subarachnoid hemorrhage in the contralateral hemisphere, while another patient developed remote hemorrhage 3 days after the procedure due to the sudden drainage of a large amount of subdural fluid over a 24-hour period. These findings suggest that catheter drainage should be carefully monitored to avoid overdrainage of CSF after burr hole trephination.
\end{abstract}

(Korean J Neurotrauma 2017;13(2):144-148)

KEY WORDS: Burr-hole trephination · Catheter drainage · Chronic subdural hematoma $\cdot$ CSF overdrainage ·

Remote hemorrhage $\cdot$ Symptomatic subdural hygroma.

\section{Introduction}

Chronic subdural hematoma (CSDH) and subdural hygroma are frequently encountered in neurosurgery, and are especially prevalent among elderly individuals. Surgical evacuation is the most common treatment approach for symptomatic patients and usually produces significant neu-

Received: April 3, 2017 / Revised: September 7, 2017

Accepted: September 7, 2017

Address for correspondence: Geun Sung Song

Department of Neurosurgery, Pusan National University Yangsan

Hospital, 20 Geumo-ro, Mulgeum-eup, Yangsan 50612, Korea

Tel: +82-55-360-2126, Fax: +82-55-360-2156

E-mail: mole83@daum.net

This work was supported by a 2-year research grant from Pusan National University.

(c) This is an Open Access article distributed under the terms of Creative Attributions Non-Commercial License (http://creativecommons. org/licenses/by-nc/4.0/) which permits unrestricted noncommercial use, distribution, and reproduction in any medium, provided the original work is properly cited. rological improvements. ${ }^{2,4)}$ Three techniques are typically used for the surgical evacuation: craniotomy, burr hole trephination, and twist-drill trephination. ${ }^{16)}$ Among these, burr hole trephination is the most popular technique due to its association with a low rate of recurrence and low patient morbidity. ${ }^{2,9,13)}$ Although controversial, several studies suggest that the use of a catheter for drainage further decreases the rate of recurrence after burr hole trephination. ${ }^{12)}$ Accordingly, burr hole trephination with catheter drainage is a popular approach for the treatment of CSDH and symptomatic subdural hygroma. However, catheter drainage is also associated with complications such as acute subdural hematoma, tension pneumocephalus, and infection. ${ }^{10,17)}$ Here, we present two rare cases of remote hemorrhages associated with catheter overdrainage after the surgical treatment of CSDH and subdural hygroma. 


\section{Case Report}

\section{Case 1}

A 53-year-old female patient presented to our emergency room with subarachnoid hemorrhage (SAH) and intracerebral hemorrhage (ICH) after undergoing burr hole trephination and catheter drainage at a local hospital. She did not exhibit any neurologic deficits and only complained of severe headache. Upon reviewing her transfer request, we discovered that she had experienced progressive headache, transient motor weakness, and mild dysarthria for a month. Preoperative neuroimaging assessments with magnetic resonance (MRI) and angiography (MRA) revealed no abnormalities other than a subdural hygroma with arachnoid cyst (Figure 1). The local hospital was unable to find any other cause for the mild dysarthria and transient motor weakness; therefore, they determined that the subdural hygroma was the cause of the patient's symptoms. She underwent evacuation of the subdural hygroma through burr hole trephination. A subdual drainage catheter (12 Fr round catheter) was placed in the subdural space, but only $130 \mathrm{cc}$ of cerebrospinal fluid (CSF) was drained over a 24-hour period and the headache persisted postoperatively and became more severe. An emergency brain computed tomography (CT) scan revealed a newly developed $\mathrm{ICH}$ and SAH in the contralateral hemisphere and $\mathrm{SAH}$ in the ipsilateral hemisphere (Figure 2). Thereafter, the local hospital transferred the patient to our emergency room for evaluation and treatment of the remote hemorrhage.

No other causes of remote hemorrhage in the contralateral hemisphere (e.g., vascular abnormalities) were identified upon reviewing the findings of the previous brain MRA examination (Figure 3). According to the local hospital's medical records, she was not on any anticoagulation and antiplatelet medications and her coagulation panel showed findings within the normal limits (prothrombin time [PT] time 10.0 seconds, PT international normalized ratio [INR] 0.93 and activated partial thromboplastin time [aPTT] 21.8 seconds). During the operation, the patient's condition was stable and blood pressure (BP) was within the normal limits. Therefore, the remote hemorrhage was suspected to be related to CSF overdrainage through the
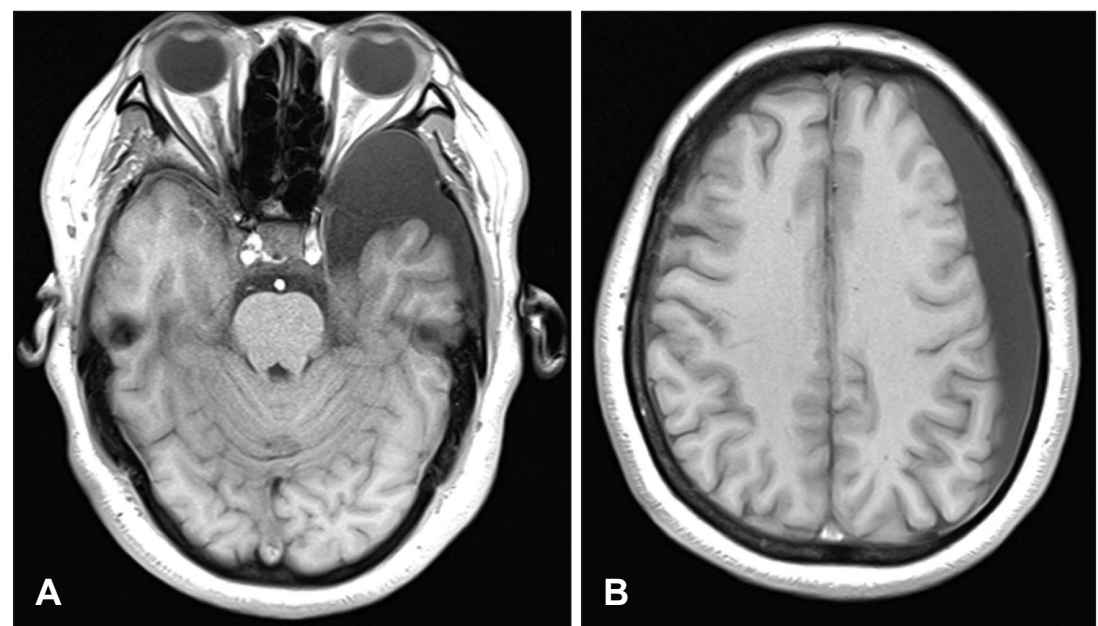

FIGURE 1. Preoperative brain magnetic resonance imaging images revealing an arachnoid cyst in the left middle cranial fossa and subdural hygroma in the left hemisphere.
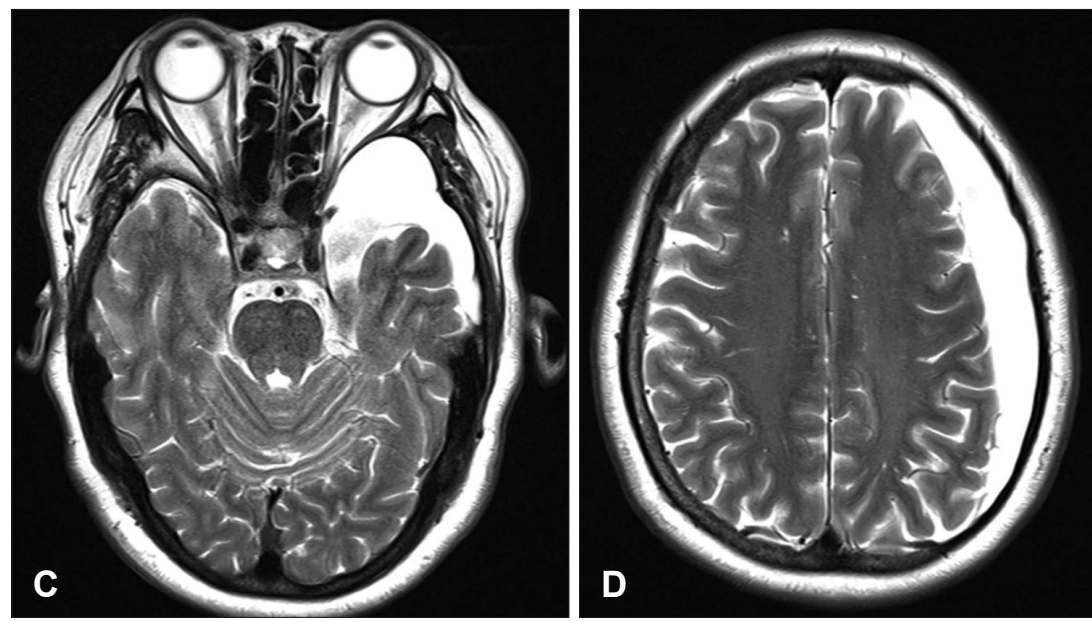
subdural drain after the burr hole trephination. We closed the subdural catheter and the patient's headache gradually subsided. Follow-up brain CT scans were performed 5 and 14 days after the catheter closure. SAH and ICH had resolved and the subdural space was refilled with CSF. One month later, the patient returned to a normal daily life.

\section{Case 2}

A 78-year-old female patient presented to our outpatient department with complaints of progressive general weakness and cognitive impairment that began a month ago. She was diagnosed with mild dementia and was admitted to a convalescence hospital but was not prescribed any medications. Despite the patient's ability to self-care at the onset of symptoms, she was unable to take care of herself or walk at the outpatient visit. Brain CT revealed bilateral subdural hygroma or bilateral CSDH with the loss of sulci markings on the cerebral hemispheres. Brain atrophy was
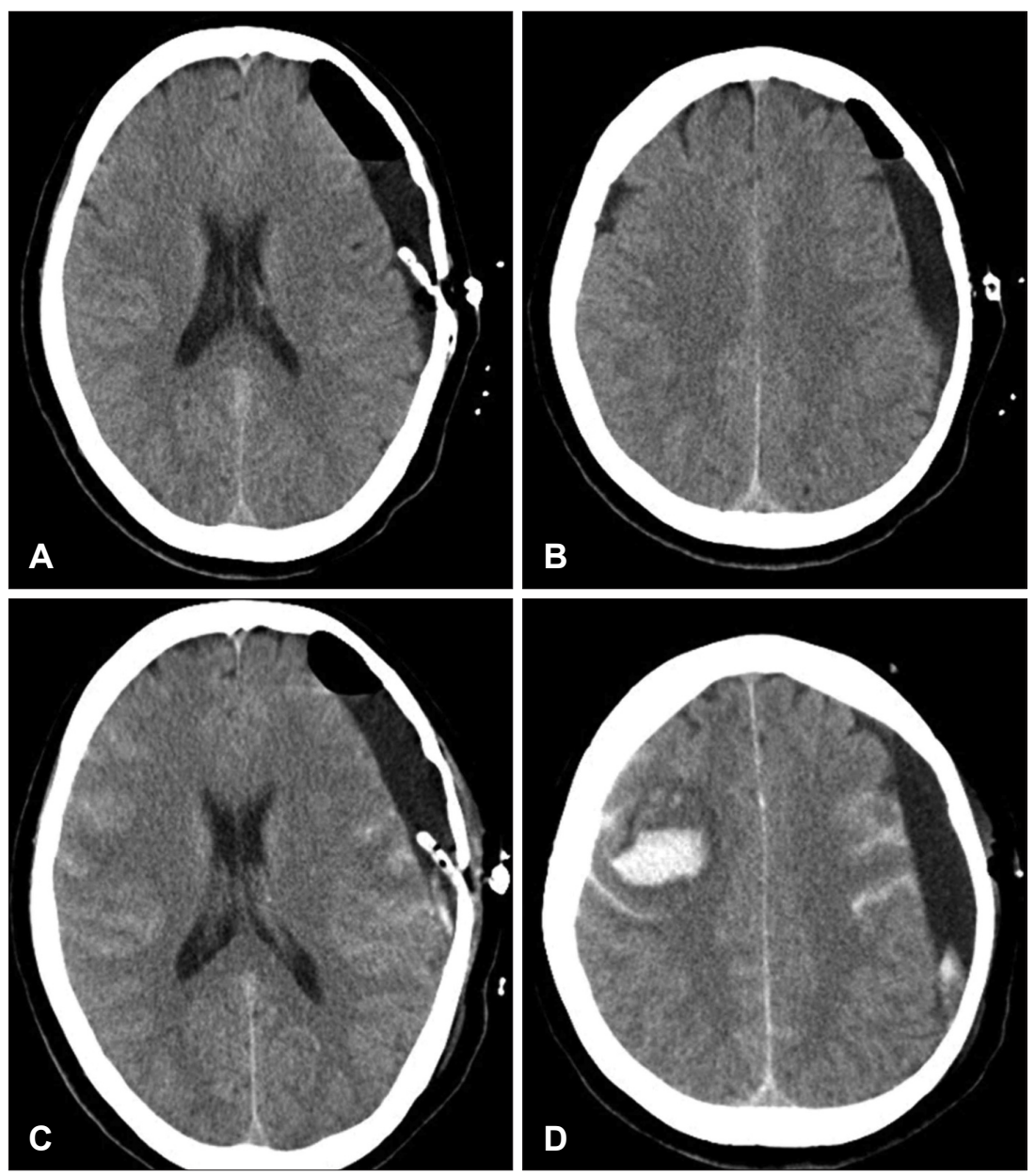

FIGURE 2. (A, B) Postoperative imaging immediately after the procedure does not reveal any specific findings, however, (C, D) newly developed subarachnoid hemorrhage and intracerebral hemorrhage are observed on computed tomography imaging the day after surgery.
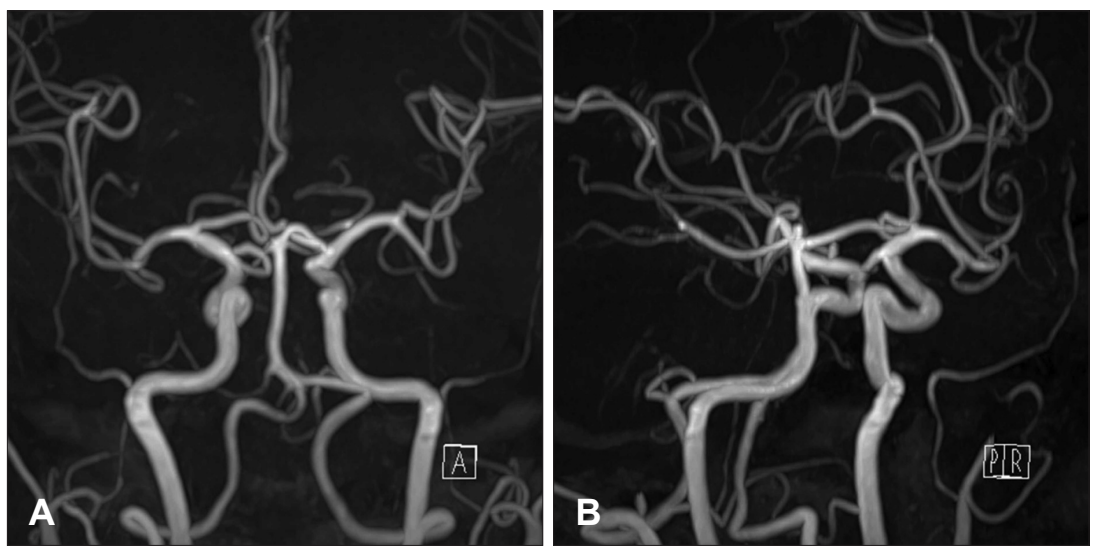

FIGURE 3. Magnetic resonance angiography does not reveal any abnormal vascular structures. 
also observed (Figure 4). Considering the rapid progression of symptoms, surgery was performed to treat the bilateral lesions. CSDH can often be misdiagnosed as a subdural hygroma on brain CT. However it was thought be $\mathrm{SDH}$ due to reddish brown fluid with clots in the operation filed. The patient underwent evacuation through 2 burr holes (one at each parietal eminence) and catheters were placed into the subdural space through both the holes to prevent further blood accumulation. The vital signs, including BP, were normal during surgery. The patient showed improvements in cognitive and motor functions postoperatively. There was no catheter drainage on the first two days postoperatively; however, $400 \mathrm{cc}$ of subdural fluid with CSF and blood was drained from the right side drainage catheter over a 24-hour period on postoperative day 3 . The patient did not exhibit any changes in the neurologic symptoms, but complained of intermittent headache. An emergency brain CT scan revealed a newly developed hemorrhage in the right subdural space and a small amount of intraventricular hemorrhage (Figure 5). CSF overdrainage was considered a possible cause of the remote hemorrhage; there- fore, we removed the drainage catheters. When we reviewed the previous images, suspected arachnoid cysts were observed in the right temporal lobe (Figure 4). One month after the surgery, a follow-up CT scan showed absorption of the hemorrhage and the area where the suspicion of the arachnoid cyst was disappeared.

\section{Discussion}

Remote hemorrhage, especially remote cerebellar hemorrhage, is a well-known postoperative complication characterized by spontaneous bleeding in the posterior fossa following supratentorial surgeries such as an aneurysm clipping, tumor resection, and lobectomies for epilepsy. $\mathrm{RCH}$ after supratentorial procedure has an incidence of approximately $0.08 \%$ to $0.6 \%{ }^{15)}$ However remote hemorrhage after burr hole evacuation and drainage of $\mathrm{CSDH}$ or subdural hygroma such as in these cases is very rare, and it is extremely rarely reported. $^{14)}$

The pathophysiology of remote hemorrhage remains unclear. Most authors agree on a multifactorial etiology of re-

FIGURE 4. Brain computed tomography imaging showing bilateral chronic subdural hematoma with the loss of sulci markings. Additionally, the right temporal lobe is smaller than the left temporal lobe, suggesting the presence of a small arachnoid cyst on the right middle cranial fossa.
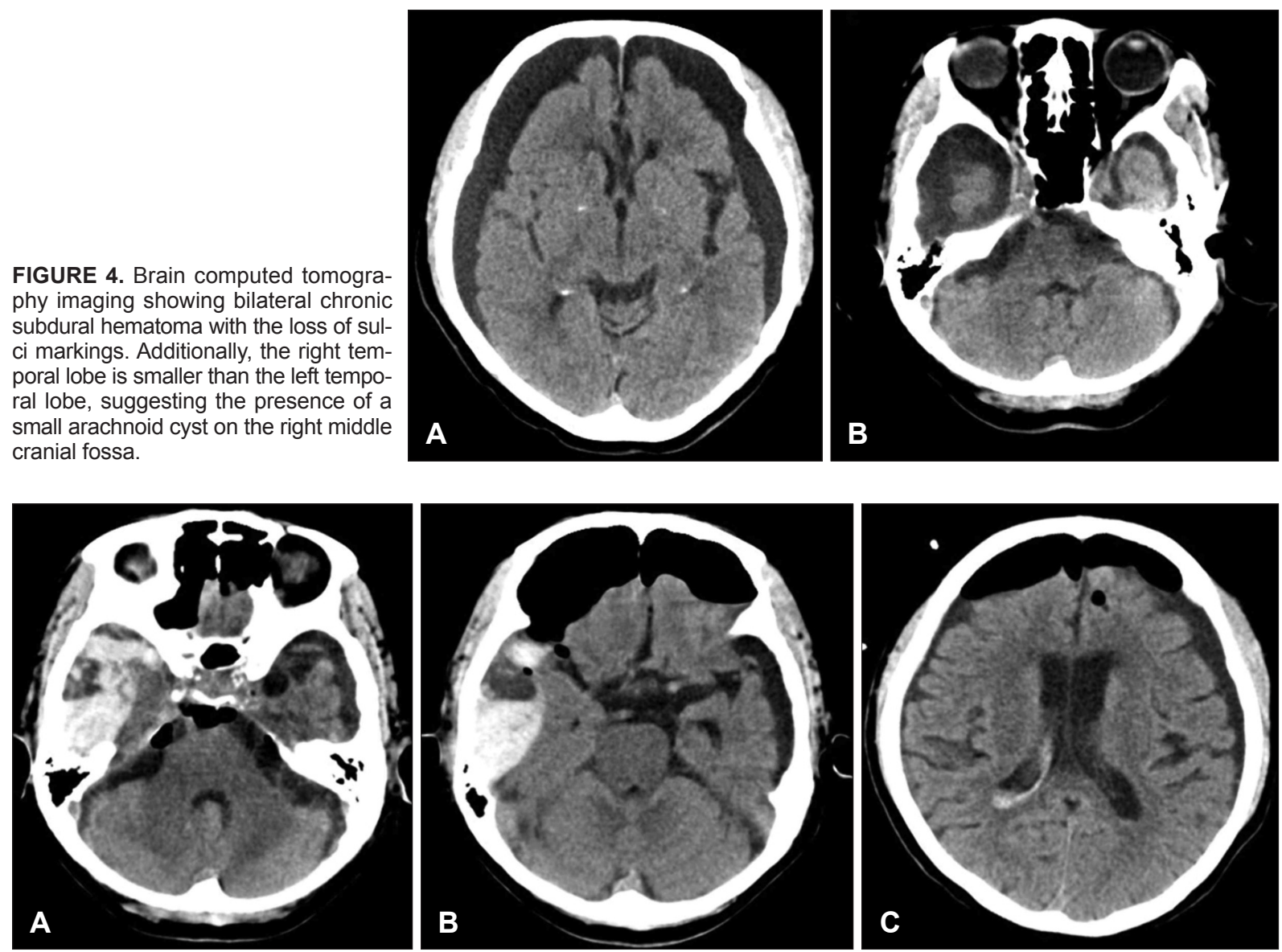

FIGURE 5. Brain computed tomography imaging 3 days after surgery. The sudden overdrainage of cerebrospinal fluid is associated with a newly developed hemorrhage in the subdural space and a small amount of intraventricular hemorrhage. 
mote hemorrhage including excessive CSF leakage, episodes of arterial hypertension, impaired venous drainage, and coagulation abnormalities. ${ }^{11)}$ Excessive CSF leakage may cause a shift in the brain parenchyma, thus, resulting in tearing and stretching of these bridging veins. Furthermore, possible mechanisms include a sudden increase in cerebral blood flow combined with failed autoregulation and damage to the parenchymal vessels secondary to the intraoperative or postoperative shifting of intracranial contents. ${ }^{3,7)}$ Cohen-Gadol ${ }^{3)}$ reported a case similar to these cases and suggested that intracranial hypotension was caused by the rapid shifting of CSF due to CSF overdrainage, and that remote hemorrhage may occur due to tension in the bridging veins or collapse of the contralateral hemisphere. Similarly, Ogasawara et al." reported that impaired vascular autoregulation was the result of long-term brain compression due to CSDH. Rapid decreases in intracranial pressure and subsequent hemodynamic changes can lead to hyperperfusion and cortical hyperemia.

Our cases is a case of remote hemorrhage after burr hole trephination with catheter drainage in CSDH or symptomatic subdural hygroma with arachnoid cyst. Arachnoid cyst is a well-known predisposing factor for CSDH following mild head trauma. ${ }^{1,5,8,14)}$ Indeed, various studies have reported the incidence of CSDH in patients with arachnoid cysts; Mori et al. $\left.{ }^{6}\right)$ reported a rate of 2.2\% (12/541 cases). However burr hole trephination with catheter drainage as a treatment for CSDH with arachnoid cyst has not been reported for remote hemorrhage.

Adding to this knowledge, our results showed that CSF overdrainage after burr hole trephination with catheter drainage in CSDH or symptomatic subdural hygroma with arachnoid cyst should be avoided to prevent remote hemorrhage.

\section{Conclusion}

In conclusion, burr hole trephination with catheter drainage is a popular procedure for the treatment of CSDH and symptomatic subdural hygroma that is rarely associated with remote hemorrhage. While the cause of remote hemorrhage was unclear in our two cases, CSF overdrainage should be considered as a possible cause. Conclusively, CSF overdrainage should be carefully avoided after catheter placement.

- The authors have no financial conflicts of interest.

\section{REFERENCES}

1) Bilginer B, Onal MB, Oguz KK, Akalan N. Arachnoid cyst associated with subdural hematoma: report of three cases and review of the literature. Childs Nerv Syst 25:119-124, 2009

2) Cenic A, Bhandari M, Reddy K. Management of chronic subdural hematoma: a national survey and literature review. Can J Neurol Sci 32:501-506, 2005

3) Cohen-Gadol AA. Remote contralateral intraparenchymal hemorrhage after overdrainage of a chronic subdural hematoma. Int J Surg Case Rep 4:834-836, 2013

4) Kudo H, Kuwamura K, Izawa I, Sawa H, Tamaki N. Chronic subdural hematoma in elderly people: present status on Awaji Island and epidemiological prospect. Neurol Med Chir (Tokyo) 32:207209, 1992

5) Kwak YS, Hwang SK, Park SH, Park JY. Chronic subdural hematoma associated with the middle fossa arachnoid cyst: pathogenesis and review of its management. Childs Nerv Syst 29:77-82, 2013

6) Mori K, Yamamoto T, Horinaka N, Maeda M. Arachnoid cyst is a risk factor for chronic subdural hematoma in juveniles: twelve cases of chronic subdural hematoma associated with arachnoid cyst. J Neurotrauma 19:1017-1027, 2002

7) Ogasawara K, Ogawa A, Okuguchi T, Kobayashi M, Suzuki M, Yoshimoto T. Postoperative hyperperfusion syndrome in elderly patients with chronic subdural hematoma. Surg Neurol 54:155159,2000

8) Page AC, Mohan D, Paxton RM. Arachnoid cysts of the middle fossa predispose to subdural haematoma formation fact or fiction? Acta Neurochir Suppl (Wien) 42:210-215, 1988

9) Ramachandran R, Hegde T. Chronic subdural hematomas-causes of morbidity and mortality. Surg Neurol 67:367-372, 2007

10) Rohde V, Graf G, Hassler W. Complications of burr-hole craniostomy and closed-system drainage for chronic subdural hematomas: a retrospective analysis of 376 patients. Neurosurg Rev 25:89-94, 2002

11) Rusconi A, Sangiorgi S, Bifone L, Balbi S. Infrequent hemorrhagic complications following surgical drainage of chronic subdural hematomas. J Korean Neurosurg Soc 57:379-385, 2015

12) Santarius T, Kirkpatrick PJ, Ganesan D, Chia HL, Jalloh I, Marcus $\mathrm{H}$, et al. Randomized controlled trial of the use of drains versus no drains after burr hole evacuation of chronic subdural hematoma: 900. Neurosurgery 65:401, 2009

13) Santarius T, Lawton R, Kirkpatrick PJ, Hutchinson PJ. The management of primary chronic subdural haematoma: a questionnaire survey of practice in the United Kingdom and the Republic of Ireland. Br J Neurosurg 22:529-534, 2008

14) Shrestha R, You C. Spontaneous chronic subdural hematoma associated with arachnoid cyst in children and young adults. Asian J Neurosurg 9:168-172, 2014

15) Sturiale CL, Rossetto M, Ermani M, Volpin F, Baro V, Milanese L, et al. Remote cerebellar hemorrhage after supratentorial procedures (part 1): a systematic review. Neurosurg Rev 39:565-573, 2016

16) Weigel R, Schmiedek P, Krauss JK. Outcome of contemporary surgery for chronic subdural haematoma: evidence based review. J Neurol Neurosurg Psychiatry 74:937-943, 2003

17) Weisse A, Berney J. Chronic subdural haematomas. Results of a closed drainage method in adults. Acta Neurochir (Wien) 127:3740, 1994 